\title{
Adaptación y Validación del Cuestionario de Compromiso Escolar en Países Iberoamericanos
}

\author{
Adaptation and Validation of the School Commitment Questionnaire in \\ Iberoamerican Countries
}

\author{
Laura Lara ${ }^{1}$, Sergio Dominguez-Lara ${ }^{2}$, Juan Miguel Gómez-Espino ${ }^{3}$, Fernando Acevedo ${ }^{4}$, \\ José Aparicio ${ }^{5}$, Mahia Saracostti ${ }^{6}$ y Edgardo Miranda-Zapata ${ }^{7}$
}

\begin{abstract}
Resumen
El objetivo de este estudio es adaptar y validar el Cuestionario de Compromiso Escolar desarrollado en Chile en adolescentes escolarizados de Colombia, Uruguay, España y Perú. Para asegurar la equivalencia cultural y lingüística en cada uno de los países, los ítems fueron en primer lugar revisados por siete expertos en educación y posteriormente por treinta adolescentes con características similares a las de la población objetivo. Los cuestionarios fueron aplicados a 1578 estudiantes en los cuatro países, a través de una plataforma informática con versiones diferenciadas para cada uno de ellos. Las propiedades psicométricas de las versiones en cada uno de los países fueron adecuadas, de este modo, los análisis de modelos de ecuaciones estructurales exploratorios mostraron que en todos los países se replicó la estructura original de tres factores (compromiso afectivo, cognitivo y conductual), y la fiabilidad fue adecuada en todos los casos.
\end{abstract}

Palabras clave: compromiso escolar, validación, cuestionario, adolescentes, estudiantes

\begin{abstract}
The objective of this study is to adapt and validate the School Engagement Questionnaire initially developed and validated in Chile with adolescents attending schools in Colombia, Uruguay, Spain and Peru. To ensure cultural and linguistic equivalence in each country, the items were reviewed by seven education experts and later by thirty adolescents with characteristics similar to those of the target population. The questionnaires were applied to 1,578 students in the four countries, through a computer platform with different versions for each of them. The psychometric properties of the versions for each country were adequate, thus, the analyses of exploratory structural equation models showed that in all the countries the original structure of three factors (affective, cognitive and behavioral engagement) was replicated, and that reliability was adequate in all cases.
\end{abstract}

Keywords: school engagement, validation, questionnaire, adolescents, students

\footnotetext{
${ }^{1}$ Doctora en Psicología. Profesora Titular. Universidad Autónoma de Chile, 5 poniente 1670, Talca, Chile. Tel.: +56712735770. Correo: 1larav@uautonoma.cl

${ }^{2}$ Doctor en Psicología. Docente-investigador. Universidad de San Martín de Porres y Universidad Privada San Juan Bautista. Av. Juan A Lavalle S/N, Lima 9, Perú. Tel.: +51 2545302. Correo: sergio.dominguez@upsjb.edu.pe

${ }^{3}$ Doctor en Sociología. Profesor Contratado Doctor. Universidad Pablo de Olavide. Facultad de Ciencias Sociales, Universidad Pablo de Olavide de Sevilla, Ctra. de Utrera 1, CP. 41013 Sevilla, España. Tel.: +34 954349348. Correo: jmgomesp@upo.es

${ }^{4}$ Doctor en Educación. Profesor Adjunto. Universidad de la República de Uruguay. Centro Universitario de Rivera, Ituzaingó 667, C.P. 40.000, Rivera, Uruguay. Tel.: +598 99866115. Correo: face@ cur.edu.uy

${ }^{5}$ Doctor en Psicología. Decano del Instituto de Estudios en Educación. Universidad del Norte. Universidad del Norte. Km.5 Vía Puerto Colombia, Barranquilla, Colombia. Tel.: +57 53509799. Correo: aparicio@ uninorte.edu.co

${ }^{6}$ Doctora en Social Welfare. Profesora Titular de la Escuela de Trabajo Social de la Facultad de Ciencias Sociales. Universidad de Valparaíso. Universidad de Valparaíso, Blanco 951, Valparaíso, Chile. Tel.: +56 998293161. Correo: mahia.saracostti@uv.cl

${ }^{7}$ Doctor en Psicología. Académico y Investigador. Núcleo Científico Tecnológico en Ciencias Sociales y Humanidades, Universidad de La Frontera. Universidad de la Frontera, Avenida Francisco Salazar, 01145. Temuco, Chile. Tel.: +56452596732. Correo: edgardo.miranda@ufrontera.cl
} 


\section{Introducción}

Una amplia literatura científica sitúa al compromiso escolar como una variable clave para el éxito académico; los resultados disponibles ponen de manifiesto que se asocia tanto con la prevención del abandono de los estudios (Tarabani Curran, Montes, \& Parcerisa, 2018; Zaff et al., 2017) como con mejores resultados académicos (Appleton, Christenson, \& Furlong, 2008). Si bien existen discrepancias en cuanto a la definición el compromiso escolar (Sinatra, Heddy, \& Lombardi, 2015), la mayoría de los autores coinciden en conceptualizarlo como una variable multidimensional, compuesta por elementos afectivos, cognitivos y conductuales (Fredricks, 2014; Lam et al., 2014). De este modo, los y las estudiantes comprometidos y comprometidas afectivamente reportan, por ejemplo, que disfrutan asistiendo a la escuela y se sienten parte de ella, sin señalar emociones negativas. El compromiso cognitivo puede observarse a través de acciones que llevan a ir más allá de los requerimientos mínimos escolares, poniendo en práctica procesos vinculados con un aprendizaje autorregulado. Finalmente, estudiantes comprometidos y comprometidas conductualmente son quienes siguen las normas escolares, como asistir regularmente a la escuela, completar las tareas escolares e inhibir conductas disruptivas en el aula.

Dada la importancia del compromiso escolar para las trayectorias educativas de los y las estudiantes, contar con instrumentos que permitan su medida posibilita detectar e intervenir de forma temprana para evitar que se produzca el abandono de los estudios. Si bien existen diversos instrumentos para su medida, la mayoría han sido creados en países anglosajones y no existe consenso sobre cuál es el más adecuado (Sinatra et al., 2015). Además, los instrumentos existentes no están exentos de problemas, entre estos, se han destacado las deficiencias en la adecuación de las propiedades psicométricas de los instrumentos que capten la multidimensionalidad del constructo (Fredricks \& McColskey, 2012) y la confusión que presentan con otras variables relacionadas (Hazel, Vazirabadi, \& Gallagher, 2013).

A este respecto, a pesar de que el compromiso escolar es concebido como una variable multidimensional, y la mayoría de los estudios coinciden en considerar que queda conformado por tres componentes (cognitivo, afectivo y conductual; (Lam et al., 2014; Lara et al., 2018; Miranda-Zapata, Lara, Navarro, Saracostti, \& deToro), algunos autores añaden un cuarto componente adicional. Por ejemplo Veiga (2016) añade un factor al que denomina personal agency, que hace referencia al interés activo y autosuficiencia del estudiante relacionado con el aprendizaje y la adquisición de conocimiento, o Wang, Fredricks, Ye, Hofkens, y Linn (2019), que añaden un componente social y ratifican una estructura bifactorial, considerando como factores el compromiso y el descompromiso, cada uno de ellos, con estos cuatro componentes. En definitiva, estos resultados están mostrando que es necesario ahondar en la multidimensionalidad del constructo.

Además, otro problema añadido, es que existen investigaciones que han utilizado instrumentos desarrollados para estudiantes de países anglosajones sin la adaptación cultural y lingüística que aseguren su correcta pertinencia a los contextos en los que se aplican. Por ejemplo, con estudiantes españoles, Rodríguez-Fernández et al. (2016) utilizan el School Engagement Measure de Fredricks, Blumenfeld, Friedel y Paris (2005) y con estudiantes dominicanos Gutiérrez, Tomás, Gómez y Moll (2019) aplican la Escala de Compromiso Escolar de Nie y Lau (2009).

De este modo, el único cuestionario desarrollado para medir el compromiso escolar en adolescentes hispanohablantes es el Cuestionario de Compromiso Escolar (CCEE) creado y validado para adolescentes chilenos por Lara et al. (2018). En el estudio en el que desarrollan y validan el cuestionario, a partir de una revisión exhaustiva de la literatura disponible, reportan una estructura de tres factores correlacionados donde se identifican los tres componentes afectivo, cognitivo y conductual, con adecuadas propiedades psicométricas. En este trabajo nos planteamos adaptar y validar el cuestionario de compromiso escolar a estudiantes escolarizados en enseñanza básica y media de Colombia, Uruguay, España y Perú.

En el caso concreto de Uruguay, pese a que el acceso a la educación media está cerca de ser universal - superior al 90\% en cada cohorte (De 
Armas \& Retamoso, 2010) -, la repetición en el primer año de este nivel es del 32\% (ANEPCODICEN, 2013) y el abandono escolar a lo largo de la educación básica sigue siendo uno de los principales problemas de su educación pública (Acevedo, 2020). Por esta razón, el énfasis de las principales políticas y programas educativos implementados en los últimos 15 años ha estado puesto en los puntos de tránsito interciclos, que es donde las tasas de abandono escolar son más preocupantes. Por ejemplo, uno de cada tres estudiantes que acceden a la educación terciaria en este país, no completan el primer tramo de este nivel (De Armas, 2017). Cuando se consideran las condiciones socioeconómicas, la deserción sube hasta el $60 \%$ en el subgrupo de los adolescentes del quintil más pobre de la población; y en el segundo tramo -educación media superior-, hasta el $90 \%$; lo cual contrasta, con el dato del $20 \%$ de deserción, del quintil más rico (Gelber, 2010).

En España, por su parte, aunque la educación tiene carácter obligatorio desde los 6 hasta los 16 años, una cuarta parte del alumnado (el 24\%) no concluye la educación secundaria obligatoria a la edad esperada (Ministerio Educación y Formación Profesional, 2017). Uno de los factores vinculado con este indicador es el de que, a los 15 años, el $28,7 \%$ de los estudiantes reporta haber repetido al menos un año de su educación básica (PISA, 2018). Este porcentaje se incrementa significativamente cuando se circunscribe el análisis al quintil más pobre de la población, pues para este subgrupo de estudiantes, es 5.6 veces más probable repetir el año, que para el quintil más rico (Politikon, 2017).

Por su parte, Perú es un país con desigualdades a todo nivel: social, étnico, económico, y regional (Cotler, 2011). Estas afectan al acceso y mantenimiento en el sistema educativo de los y las estudiantes, de este modo, aspectos como el sexo, idioma del hogar, ocupación de los padres, entre otros, han mostrado influir en su desempeño escolar (Muelle, 2016). Gran parte del fracaso escolar en las pruebas internacionales (e.g., PISA) se debe al contexto sociocultural de la familia del estudiante (OCDE, 2007). En ese sentido, en el lustro 2005-2010 el porcentaje de fracaso escolar fue alrededor del $30 \%$ en Perú (Latorre, 2018). Estas evidencias de las desigualdades sociales comunes, sugieren la necesidad de abandonar planteamientos dicotómicos sobre el fracaso-éxito escolar, y abordar el fenómeno educativo en toda su complejidad. Por esta razón, una perspectiva centrada en los déficits que conducen a la desafección (desvinculación o desenganche) escolar (Enguita, 2011) debe complementarse con la indagación de los factores que conducen al compromiso escolar. Sólo así, se podrán desarrollar políticas educativas que promuevan para el estudiantado trayectorias educativas completas y de calidad.

Finalmente, en el caso de Colombia, tan solo se cuenta con un antecedente que se ha centrado en el estudio del compromiso escolar, en este caso en educación básica y con técnicas de medición cualitativas (Pineda-Báez, Hennig, \& Vargas, 2019). En Colombia, la preocupación de las políticas públicas educativas ha estado centrada en la última década en promover el acceso y permanencia de los niños y niñas al sistema educativo. Este énfasis, ha traído como efecto positivo un aumento en la cobertura en educación básica primaria y secundaria (Banco Mundial, 2020). Sin embargo, el derecho a la educación no debe entenderse sólo como la oportunidad de acceso al sistema escolar, sino que las políticas públicas que se implementen en un país deben orientarse hacia el progresivo alcance de una educación de calidad para todos y todas. Sin embargo, los resultados de las pruebas PISA ponen de manifiesto que Colombia muestra aún pocos avances en términos de calidad (OCDE 2016; OCDE, 2019), así como las dificultades que persisten para superar las inequidades educativas existentes. Por ejemplo, sólo un $10 \%$ de los estudiantes de menor nivel socioeconómico se clasificaron en el nivel superior en lectura, en la prueba PISA 2018 (OCDE, 2019). Es necesario avanzar hacia una visión integradora de la comprensión del éxito escolar a partir de un seguimiento individualizado de los factores que afectan el aprendizaje de cada estudiante, posicionándose la posibilidad de analizar el compromiso escolar con instrumentos válidos y confiables como un aporte que permitiría completar esta visión y poder aplicar políticas públicas más efectivas.

A partir de todo lo expuesto, el objetivo de este estudio es adaptar y validar el cuestionario de 
compromiso escolar a estudiantes escolarizados en enseñanza básica y media de Colombia, Perú, Uruguay y España. Se espera que el cuestionario presente adecuados índices psicométricos en cada uno de los países, replicándose la estructura factorial de tres factores relacionados (compromisos afectivo, cognitivo y conductual) y presente adecuados índices de confiabilidad.

\section{Método}

\section{Participantes}

En este estudio participaron 1578 estudiantes (55.4\% chicos) con edades comprendidas entre los 12 y los 19 años escolarizados en educación básica y media en Colombia, Uruguay, España y Perú. En Colombia se evaluaron 438 estudiantes (55.9\% chicos) que cursaban $7^{\circ}, 8^{\circ}$ y $9^{\circ}$ grado de educación básica secundaria, y $10^{\circ}$ y $11^{\circ}$ de educación media $(21.7 \%, 17.1 \%, 22.4 \%, 22.1 \%$ y $16.7 \%$, respectivamente). En Uruguay participaron 391 estudiantes $(67.5 \%$ chicos), que cursaban $1^{\circ}, 2^{\circ}$ y $3^{\circ}$ año del ciclo básico de media y $1^{\circ}$ y $2^{\circ}$ de bachillerato $(20.7 \%, 20.7 \%, 21 \%$, $18.7 \%$ y $18.9 \%$, respectivamente). En España participaron 394 estudiantes (52.3\% chicos), que cursaban $1^{\circ}, 2^{\circ}, 3^{\circ}$ y $4^{\circ}$ grado de enseñanza secundaria obligatoria y $1^{\circ}$ de bachillerato $(19.8 \%$, $20.3 \%, 18.3 \%, 23.6 \%$ y $17.5 \%$, respectivamente). Finalmente, en Perú participaron 355 estudiantes ( $45.1 \%$ chicos), que cursaban $1^{\circ}, 2^{\circ}, 3^{\circ}, 4^{\circ}$ y $5^{\circ}$ de educación secundaria $(18.6 \%, 18.6 \%, 22.3 \%$, $21.1 \%$ y $19.4 \%$, respectivamente).

\section{Instrumentos}

Cuestionario de Compromiso Escolar (CCE; Lara et al., 2018). Es un instrumento de autoinforme que mide las tres dimensiones del compromiso escolar: afectivo (10 ítems), cognitivo (12 ítems) y conductual (7 ítems). Está compuesto por 29 ítems escalados en formato Likert que va de 1 (nunca o casi nunca) a 5 (siempre o casi siempre). Fue desarrollado con adolescentes en Chile, y la evidencia psicométrica fue favorable tanto para su estructura interna de tres factores como de consistencia interna (alfa ordinal de .83 para el compromiso afectivo, .86 para el conductual y .87 para el cognitivo; y de .95 para la escala total).
La adaptación y ajuste del cuestionario, originalmente desarrollado y validado con estudiantes chilenos (CCE, Lara et al., 2018), se llevó a cabo a través del juicio de expertos y grupos de discusión con estudiantes, en cada uno de los cuatro países. Con el objetivo de minimizar posibles sesgos derivados de interpretaciones inadecuadas de los ítems, todo el proceso fue acompañado por una de las creadoras del instrumento. En todo momento, se trató de mantener el significado original de los ítems, solo realizando las modificaciones necesarias para su adecuada comprensión en cada contexto.

En cada uno de los países se contactó por email a siete expertos de áreas relacionadas con la educación (psicología educativa y ciencias de la educación), todos ellos académicos que trabajaban con niños/as y adolescentes. Su labor consistió en revisar los cuestionarios y realizar juicios de adecuación de los ítems, indicando, cuando era necesario, las alternativas posibles para su redacción.

Una vez recibidas las respuestas de los siete expertos, los cambios sugeridos fueron analizados por los investigadores. Como regla general, se dejaron igual aquellos ítems en los que ningún experto señaló problemas de comprensión, y se reescribieron aquellos ítems en los que todos los expertos sugirieron modificaciones. Se propusieron cambios provisionales en aquellos ítems en los que más de un experto señaló problemas, para ser posteriormente probados en los grupos de discusión con estudiantes, presentándoles tanto la redacción original, como la sugerida por los expertos.

Unificados los cambios propuestos por los expertos y las expertas, se llevaron a cabo 20 grupos de discusión, en los que participaron 120 adolescentes; para verificar con ellos su comprensión de los cambios propuestos por los expertos. En cada país se realizaron 5 grupos de discusión (uno por cada grado académico), de 6 estudiantes cada uno, 3 chicos y 3 chicas. Los encuentros se realizaron en los centros educativos donde los estudiantes estaban matriculados. Fueron liderados por el investigador local de cada país, con el acompañamiento de la investigadora chilena. 
Tabla 1. Solución factorial muestra colombiana

\begin{tabular}{|c|c|c|c|c|c|c|c|c|c|}
\hline & & \multicolumn{4}{|c|}{ Inicial } & \multicolumn{4}{|c|}{ Final } \\
\hline & & AFE & $\mathrm{COG}$ & $\mathrm{CON}$ & ISF & $\mathrm{AFE}$ & $\mathrm{COG}$ & $\mathrm{CON}$ & ISF \\
\hline 1 & Siento que soy parte del colegio & .800 & -.022 & -.113 & .970 & .722 & .069 & -.048 & .980 \\
\hline 5 & Puedo ser yo mismo(a) en este colegio & .488 & -.065 & .034 & .967 & .452 & -.013 & .049 & .981 \\
\hline 7 & $\begin{array}{l}\text { La mayoría de las cosas que aprendo en el } \\
\text { colegio son útiles }\end{array}$ & .419 & .164 & .277 & .469 & & & & \\
\hline 8 & $\begin{array}{l}\text { La mayoría de los profesores se preocupan de } \\
\text { que la materia que aprendamos sea útil }\end{array}$ & .460 & .091 & .224 & 689 & & & & \\
\hline 12 & Me siento orgulloso/a de estar en este colegio & .897 & -.124 & .004 & .972 & .852 & -.036 & .026 & .996 \\
\hline 15 & $\begin{array}{l}\text { Para mí es muy importante lo que hacemos en } \\
\text { el colegio }\end{array}$ & .439 & .294 & .189 & .441 & & & & \\
\hline 19 & Me tratan con respeto en este colegio & .519 & .103 & .037 & .937 & .420 & .159 & .092 & .762 \\
\hline 22 & $\begin{array}{l}\text { Lo que aprendo en clase es importante para } \\
\text { conseguir mis metas en el futuro }\end{array}$ & .294 & .200 & .440 & .426 & & & & \\
\hline 27 & Siento que soy importante para el colegio & .721 & .098 & -.387 & .672 & & & & \\
\hline 29 & Me siento bien en este colegio & .872 & -.128 & .012 & .968 & .868 & -.067 & .030 & .989 \\
\hline 2 & $\begin{array}{l}\text { Antes de un examen, planeo cómo estudiar la } \\
\text { asignatura. }\end{array}$ & .022 & .474 & -.184 & .809 & -.014 & .498 & -.144 & .886 \\
\hline 6 & $\begin{array}{l}\text { Utilizo distintos recursos (como internet o } \\
\text { libros) para reforzar la información entregada } \\
\text { por el profesor }\end{array}$ & .070 & .568 & -.205 & .814 & .004 & .636 & -.145 & .927 \\
\hline 10 & $\begin{array}{l}\text { Cuando estoy haciendo alguna actividad, me } \\
\text { preocupo de entender todo lo posible. }\end{array}$ & -.086 & .586 & .141 & .890 & -.109 & .565 & .183 & .815 \\
\hline 13 & $\begin{array}{l}\text { Después de un examen pienso si mis } \\
\text { respuestas fueron las correctas }\end{array}$ & .111 & .499 & .048 & .917 & .033 & .524 & .098 & .944 \\
\hline 14 & $\begin{array}{l}\text { Sé qué estrategias y hábitos de estudio tengo } \\
\text { que cambiar para mejorar y obtener mejores } \\
\text { calificaciones }\end{array}$ & .220 & .406 & -.007 & .688 & .104 & .455 & .057 & .905 \\
\hline 17 & $\begin{array}{l}\text { Cuando comienzo una tarea, pienso en las } \\
\text { cosas que ya sé sobre el tema porque eso me } \\
\text { ayuda a comprender mejor }\end{array}$ & -.008 & .638 & .049 & .991 & -.098 & .630 & .136 & .901 \\
\hline 18 & $\begin{array}{l}\text { Cuando estudio, anoto palabras nuevas, dudas } \\
\text { o ideas importantes }\end{array}$ & -.256 & .518 & -.195 & .586 & & & & \\
\hline 20 & $\begin{array}{l}\text { Para mí es importante lograr entender bien las } \\
\text { tareas y las asignaturas }\end{array}$ & .079 & .494 & .366 & .536 & & & & \\
\hline 21 & $\begin{array}{l}\text { Sé cómo utilizar diferentes estrategias para } \\
\text { realizar bien mis tareas (como, por ejemplo, } \\
\text { planear el trabajo, destacar ideas principales, } \\
\text { discutir en grupos, etc.) }\end{array}$ & .109 & .528 & -.014 & .938 & .017 & .553 & .065 & .978 \\
\hline 24 & $\begin{array}{l}\text { Después de terminar mis tareas reviso si están } \\
\text { bien }\end{array}$ & .031 & .673 & -.122 & .95 & -.013 & .646 & -.052 & .990 \\
\hline 25 & $\begin{array}{l}\text { Cuando termino una tarea, pienso si he } \\
\text { conseguido lo que me había propuesto }\end{array}$ & .156 & .590 & -.203 & .763 & .131 & .597 & -.160 & .840 \\
\hline 26 & $\begin{array}{l}\text { Presto atención a la opinión de los profesores } \\
\text { sobre mis trabajos. }\end{array}$ & .028 & .563 & .347 & .631 & & & & \\
\hline 3 & Me escapo de clase o me echo la leva (r) & .168 & .015 & .750 & .929 & .046 & .045 & .812 & .991 \\
\hline 4 & Salgo del salón de clase, sin pedir permiso (r) & .088 & -.004 & .718 & .978 & .030 & -.036 & .755 & .994 \\
\hline 9 & Llego tarde a clase (r) & .119 & .043 & .670 & .949 & .050 & .027 & .716 & .991 \\
\hline 11 & $\begin{array}{l}\text { Han citado a mis padres o acudientes por mi } \\
\text { mal comportamiento }(r)\end{array}$ & .130 & .036 & .734 & .951 & .052 & .041 & .775 & .989 \\
\hline 16 & Me porto bien en la clase & -.062 & .467 & .342 & .548 & & & & \\
\hline 23 & $\begin{array}{l}\text { Discuto o peleo con mis compañeros/as en el } \\
\text { salón de clase (r) }\end{array}$ & .093 & .015 & .536 & .955 & .004 & .039 & .600 & .994 \\
\hline 28 & $\begin{array}{l}\text { Me mandan a la oficina del rector o del } \\
\text { coordinador por mi mal comportamiento (r) }\end{array}$ & .086 & -.037 & .811 & .980 & -.016 & -.039 & .871 & .996 \\
\hline
\end{tabular}

Nota. AFE=Afectivo; $\mathrm{COG}=$ Cognitivo; $\mathrm{CON}=$ Conductual; $(\mathrm{r})=$ ítem reverso

\section{Procedimiento}

La recolección de datos se realizó de forma online, en cada uno de los centros educativos participantes de Perú, Colombia, Uruguay y España. Los centros educativos se encontraban ubicados en zonas urbanas y fueron de gestión pública excepto en el caso de Perú que fueron de gestión privada. Las versiones adaptadas de los cuestionarios (a partir de los juicios de expertos y grupos de discusión con estudiantes), para cada uno de los cuatro países participantes, fueron alojadas en una plataforma informática 
Tabla 2. Solución factorial muestra uruguaya

\begin{tabular}{|c|c|c|c|c|c|c|c|c|c|}
\hline & \multicolumn{4}{|c|}{ Inicial } & \multicolumn{4}{|c|}{ Final } \\
\hline & & $\mathrm{AFE}$ & $\mathrm{COG}$ & $\mathrm{CON}$ & ISF & $\mathrm{AFE}$ & $\mathrm{COG}$ & $\mathrm{CON}$ & ISF \\
\hline 1 & Siento que soy parte del liceo & .730 & -.233 & -.053 & .858 & .629 & -.031 & -.072 & .977 \\
\hline 5 & Puedo ser yo mismo(a) en este liceo & .630 & -.227 & .012 & .833 & .690 & -.129 & .019 & .949 \\
\hline 7 & $\begin{array}{l}\text { La mayoría de las cosas que aprendo en el liceo } \\
\text { son útiles }\end{array}$ & .300 & .660 & -.081 & .740 & & & & \\
\hline 8 & $\begin{array}{l}\text { La mayoría de los profesores se preocupan de } \\
\text { que la materia que aprendamos sea útil }\end{array}$ & .313 & .620 & -.181 & .629 & & & & \\
\hline 12 & Siento orgullo de estar en este liceo & .513 & .449 & -.200 & .396 & & & & \\
\hline 15 & $\begin{array}{l}\text { Para mí es muy importante lo que hacemos en } \\
\text { el liceo }\end{array}$ & .301 & .728 & -.066 & .781 & & & & \\
\hline 19 & Me tratan con respeto en este liceo & .561 & .032 & .114 & .937 & .546 & .117 & .142 & .847 \\
\hline 22 & $\begin{array}{l}\text { Lo que aprendo en clase es importante para } \\
\text { conseguir mis metas futuras }\end{array}$ & .182 & .833 & -.116 & .906 & & & & \\
\hline 27 & Siento que soy importante para el liceo & .676 & -.084 & -.092 & .951 & .463 & .147 & -.098 & .810 \\
\hline 2 & $\begin{array}{l}\text { Antes de una prueba, organizo cómo estudiar la } \\
\text { materia }\end{array}$ & .377 & .221 & .175 & .468 & & & & \\
\hline 6 & $\begin{array}{l}\text { Utilizo distintos recursos (como internet o } \\
\text { libros) para buscar información complementaria } \\
\text { a la entregada por el profesor }\end{array}$ & .314 & .322 & .121 & .399 & & & & \\
\hline 10 & $\begin{array}{l}\text { Cuando estoy haciendo alguna actividad, me } \\
\text { preocupo de entender todo lo posible. }\end{array}$ & .335 & .396 & .290 & .180 & & & & \\
\hline 13 & $\begin{array}{l}\text { Después de una prueba pienso si las respuestas } \\
\text { fueron las correctas }\end{array}$ & .190 & .556 & .105 & .804 & .030 & .712 & -.024 & .996 \\
\hline 14 & $\begin{array}{l}\text { Sé qué hábitos de estudio tengo que cambiar } \\
\text { para mejorar y obtener mejores calificaciones. }\end{array}$ & .195 & .452 & .107 & .713 & .103 & .546 & .017 & .948 \\
\hline 17 & $\begin{array}{l}\text { Cuando comienzo una tarea, pienso en las cosas } \\
\text { que ya sé sobre el tema porque eso me ayuda a } \\
\text { comprender mejor }\end{array}$ & .150 & .705 & .170 & .859 & -.037 & .873 & .026 & .996 \\
\hline 18 & $\begin{array}{l}\text { Cuando estudio, anoto palabras nuevas, dudas o } \\
\text { ideas importantes }\end{array}$ & .194 & .502 & .108 & .758 & -.075 & .772 & -.054 & .979 \\
\hline 20 & $\begin{array}{l}\text { Para mí es importante lograr entender bien las } \\
\text { tareas y la materia }\end{array}$ & .193 & .621 & .211 & .738 & .038 & .717 & .116 & .958 \\
\hline 21 & $\begin{array}{l}\text { Sé cómo utilizar diferentes técnicas para } \\
\text { realizar bien mis tareas (como, por ejemplo, } \\
\text { organizar el trabajo, destacar ideas principales, } \\
\text { discutir en grupos, etc.) }\end{array}$ & .332 & .412 & .261 & .254 & & & & \\
\hline 24 & $\begin{array}{l}\text { Después de terminar mis tareas reviso si están } \\
\text { bien }\end{array}$ & .371 & .389 & .135 & .413 & & & & \\
\hline 25 & $\begin{array}{l}\text { Cuando finalizo una tarea, pienso si he } \\
\text { conseguido el objetivo que me había propuesto }\end{array}$ & .235 & .667 & .098 & .813 & .042 & .857 & -.030 & .995 \\
\hline 26 & $\begin{array}{l}\text { Pongo atención a los comentarios que los } \\
\text { profesores hacen sobre mis trabajos. }\end{array}$ & .412 & .304 & .230 & .327 & & & & \\
\hline 3 & Me escapo de clase o me hago la rabona (r) & -.133 & .073 & .695 & .932 & -.063 & .003 & .680 & .987 \\
\hline 4 & Salgo sin pedir permiso de la clase (r) & -.088 & .373 & .411 & .47 & & & & \\
\hline 9 & Llego tarde a clase (r) & -.052 & .075 & .452 & .941 & .019 & .053 & .432 & .975 \\
\hline 11 & $\begin{array}{l}\text { Mis padres o tutores han sido citados por mi } \\
\text { mala conducta }(r)\end{array}$ & -.084 & -.007 & .827 & .985 & .029 & -.069 & .832 & .988 \\
\hline 16 & Me porto bien en clase & .018 & .020 & .756 & .998 & .089 & -.005 & .721 & .978 \\
\hline 23 & Peleo con mis compañeros en la clase (r) & -.114 & .233 & .587 & .760 & -.028 & .169 & .584 & .884 \\
\hline 28 & $\begin{array}{l}\text { Me mandan a la adscripción o a la dirección por } \\
\text { mi mala conducta }(r)\end{array}$ & -.186 & .119 & .826 & .900 & -.039 & -.051 & .874 & .992 \\
\hline
\end{tabular}

Nota. AFE=Afectivo; COG=Cognitivo; CON=Conductual; (r)=ítem reverso; ítem 29 fue un caso Heywood (carga factorial >1).

(Saracostti, Lara, \& Miranda-Zapata, 2019) de forma diferenciada, de manera que cada país podía acceder sólo a su propia versión del cuestionario. Los cuestionarios mantuvieron equivalencia en su contenido, solo variando en la redacción y el vocabulario (ver Tablas 1, 2, 3 y 4).

El manejo de la plataforma y el control de acceso fue responsabilidad del equipo chileno, que contó con una empresa contratada para tal fin. En cada país, los y las participantes completaron los cuestionarios en los computadores de los centros educativos, durante su horario lectivo. Los acompañaban el investigador local y el profesor o profesora responsable del curso, con el fin de generar un ambiente de confianza y resolver sus dudas. Se cumplieron los requerimientos éticos de cada país, contando siempre con la autorización de los centros educativos, los consentimientos de los tutores y/o las tutoras legales y los asentimientos de los y las estudiantes. El estudio recibió la 
Tabla 3. Solución factorial muestra española

\begin{tabular}{|c|c|c|c|c|c|c|c|c|c|}
\hline & \multicolumn{4}{|c|}{ Inicial } & \multicolumn{4}{|c|}{ Final } \\
\hline & & AFE & $\mathrm{COG}$ & $\mathrm{CON}$ & ISF & AFE & $\mathrm{COG}$ & $\mathrm{CON}$ & ISF \\
\hline 1 & Siento que soy parte del instituto & .683 & -.198 & .136 & .836 & .608 & -.065 & .152 & .898 \\
\hline 5 & Puedo ser yo mismo(a) en este instituto & .635 & -.225 & .054 & .829 & .602 & -.088 & .054 & .957 \\
\hline 7 & $\begin{array}{l}\text { La mayoría de las cosas que aprendo en el instituto me } \\
\text { sirven para algo }\end{array}$ & .560 & .265 & .025 & .74 & & & & \\
\hline 8 & $\begin{array}{l}\text { La mayoría del profesorado se preocupa de que la } \\
\text { materia que aprendamos nos sirva para algo }\end{array}$ & .508 & .204 & .050 & .789 & & & & \\
\hline 12 & Me siento orgulloso/a de estar en este instituto & .793 & .002 & -.274 & .845 & .736 & .099 & -.239 & .838 \\
\hline 15 & $\begin{array}{l}\text { Para mí es muy importante lo que hacemos en el } \\
\text { instituto }\end{array}$ & .525 & .407 & -.099 & .513 & & & & \\
\hline 19 & Me tratan con respeto en este instituto & .641 & -.069 & .149 & .908 & .581 & .048 & .162 & .885 \\
\hline 22 & Lo que aprendo en clases es importante para mi futuro & .585 & .213 & 125 & .776 & & & & \\
\hline 27 & Siento que soy importante para el instituto & .707 & -.043 & -.364 & .705 & & & & \\
\hline 29 & Me siento bien en este instituto & .835 & -.084 & -.053 & .979 & .868 & .017 & -.067 & .991 \\
\hline 2 & Antes de un examen, organizo cómo estudiar la materia & -.105 & .696 & -.056 & .957 & -.065 & .665 & .044 & .979 \\
\hline 6 & $\begin{array}{l}\text { Utilizo distintos recursos (como internet o libros) para } \\
\text { buscar información complementaria a la que da el } \\
\text { profesor/a }\end{array}$ & .039 & .359 & -.024 & .976 & -.022 & .392 & .083 & .932 \\
\hline 10 & $\begin{array}{l}\text { Cuando estoy haciendo alguna actividad, me preocupo } \\
\text { de entender todo lo posible. }\end{array}$ & .047 & .55 & .358 & .603 & & & & \\
\hline 13 & $\begin{array}{l}\text { Después de un examen pienso si mis respuestas fueron } \\
\text { las correctas }\end{array}$ & .118 & .389 & .318 & .452 & & & & \\
\hline 14 & $\begin{array}{l}\text { Sé que tengo que hacer para mejorar y obtener mejores } \\
\text { notas }\end{array}$ & .367 & -.077 & .427 & .489 & & & & \\
\hline 17 & $\begin{array}{l}\text { Cuando comienzo una tarea, pienso en las cosas que ya } \\
\text { sé sobre el tema porque eso me ayuda a comprender } \\
\text { mejor }\end{array}$ & .094 & .668 & .072 & .954 & .053 & .666 & .187 & .884 \\
\hline 18 & $\begin{array}{l}\text { Cuando estudio, apunto palabras nuevas, dudas o ideas } \\
\text { importantes }\end{array}$ & -.025 & .797 & -.326 & .794 & -.055 & .791 & -.195 & .909 \\
\hline 20 & $\begin{array}{l}\text { Para mí es importante lograr entender bien las tareas y } \\
\text { la materia }\end{array}$ & .158 & .566 & .377 & .529 & & & & \\
\hline 21 & $\begin{array}{l}\text { Sé cómo utilizar diferentes técnicas para realizar bien } \\
\text { mis tareas (como, por ejemplo, planificar el trabajo, } \\
\text { destacar ideas principales, discutir en grupos, etc.) }\end{array}$ & .131 & .453 & .139 & .774 & .088 & .482 & .198 & .754 \\
\hline 24 & Después de terminar mis tareas reviso si están bien & .044 & .717 & -.196 & .893 & .013 & .720 & -.093 & .975 \\
\hline 25 & $\begin{array}{l}\text { Cuando finalizo una tarea, pienso si he conseguido el } \\
\text { objetivo que me había propuesto }\end{array}$ & .124 & .684 & -.166 & .874 & .047 & .701 & -.030 & .991 \\
\hline 26 & $\begin{array}{l}\text { Pongo atención a los comentarios que los profesores/as } \\
\text { hacen sobre mis trabajos. }\end{array}$ & .172 & .426 & .426 & .387 & & & & \\
\hline 3 & Me salto las clases o hago rabonas (r) & .094 & -.033 & .870 & .981 & .048 & -.049 & .894 & .991 \\
\hline 4 & Salgo sin pedir permiso de la clase (r) & .031 & .041 & .860 & .995 & -.054 & .021 & .912 & .994 \\
\hline 9 & Llego tarde a clase $(r)$ & .014 & .086 & .673 & .975 & -.004 & .092 & .703 & .975 \\
\hline 11 & $\begin{array}{l}\text { Mis padres o tutores legales han sido citados por mi } \\
\text { mala conducta (r) }\end{array}$ & -.024 & .128 & .791 & .961 & -.020 & .06 & .811 & .991 \\
\hline 16 & Me porto bien en clase & -.017 & .437 & .512 & .517 & & & & \\
\hline 23 & Peleo o discuto con mis compañeros/as en la clase $(r)$ & -.039 & .067 & .552 & .971 & .012 & .036 & .539 & .993 \\
\hline 28 & $\begin{array}{l}\text { Me mandan al despacho del director/a o del jefe de } \\
\text { estudios por mi mala conducta (r) }\end{array}$ & .096 & -.017 & .864 & .981 & .103 & -.027 & .858 & .977 \\
\hline
\end{tabular}

Nota. AFE=Afectivo; $\mathrm{COG}=$ Cognitivo; $\mathrm{CON}=$ Conductual; $(\mathrm{r})=$ ítem revers

aprobación del Comité de Ética de la Universidad de la Frontera (Chile) y de la Comisión Nacional de Investigación Científica y Tecnológica de Chile.

\section{Análisis de datos}

Para analizar la estructura factorial de los cuestionarios en cada uno de los países, se generaron modelos exploratorios de ecuaciones estructurales (ESEM, Asparouhov, \& Muthen, 2009) de forma separada para cada una de las muestras. Mediante el programa Mplus v. 7
(Muthen \& Muthen, 1998-2015), se hizo la estimación WLSMV usando las correlaciones policóricas. Se especificó la rotación target oblicua ( $\varepsilon=.05$; Asparouhov \& Muthen, 2009) que consistió en estimar libremente los ítems que pertenecían teóricamente a cada factor y se especificaron como cercanos a cero $(\sim 0)$, los ítems que no pertenecían a dicho factor. El modelo fue evaluado en cada país mediante la combinación de los índices de bondad de ajuste del modelo y un enfoque exploratorio, para la conservación de los ítems. 
Tabla 4. Solución factorial muestra peruana

\begin{tabular}{|c|c|c|c|c|c|c|c|c|c|}
\hline & & & Inic & & & & $\mathrm{Fil}$ & & \\
\hline & & AFE & $\mathrm{COG}$ & $\mathrm{CON}$ & ISF & $\mathrm{AFE}$ & $\mathrm{COG}$ & $\mathrm{CON}$ & ISF \\
\hline 1 & Siento que soy parte del colegio & .754 & -.094 & -.002 & .977 & .799 & -.088 & .010 & .982 \\
\hline 5 & Puedo ser yo mismo(a) en este colegio & .565 & -.134 & -.100 & .879 & .573 & -.119 & -.067 & .920 \\
\hline 7 & $\begin{array}{l}\text { La mayoría de las cosas que aprendo en el colegio } \\
\text { son útiles }\end{array}$ & .542 & 162 & .160 & .775 & & & & \\
\hline 8 & $\begin{array}{l}\text { La mayoría de los profesores se preocupan de que el } \\
\text { curso que aprendamos sea útil }\end{array}$ & .639 & -.011 & .112 & .955 & .496 & .103 & 116 & .866 \\
\hline 12 & Siento orgullo de estar en este colegio & .836 & .029 & -.160 & .946 & .774 & .102 & -.143 & .927 \\
\hline 15 & $\begin{array}{l}\text { Para mí es muy importante lo que hacemos en el } \\
\text { colegio }\end{array}$ & .445 & .394 & .057 & .497 & & & & \\
\hline 19 & Me tratan con respeto en este colegio & .416 & .133 & .199 & 632 & .450 & .138 & .205 & 657 \\
\hline 22 & $\begin{array}{l}\text { Lo que aprendo en clases es importante para } \\
\text { conseguir mis metas futuras }\end{array}$ & .465 & .300 & .125 & .546 & & & & \\
\hline 27 & Siento que el colegio se interesa por mi & .766 & .038 & -.073 & .983 & .737 & .079 & -.028 & .981 \\
\hline 2 & $\begin{array}{l}\text { Antes de un examen, planifico cómo estudiar el } \\
\text { curso }\end{array}$ & .002 & .633 & -.027 & .997 & -.071 & .694 & -.048 & .977 \\
\hline 6 & $\begin{array}{l}\text { Utilizo distintos recursos (como internet o libros) } \\
\text { para buscar información complementaria entregada } \\
\text { por el profesor }\end{array}$ & .04 & .386 & -.019 & .981 & .012 & .407 & -.033 & .989 \\
\hline 10 & $\begin{array}{l}\text { Cuando estoy haciendo alguna actividad, me } \\
\text { preocupo de entender todo lo posible }\end{array}$ & .197 & .557 & .04 & .832 & .136 & .608 & .022 & .928 \\
\hline 13 & $\begin{array}{l}\text { Después de un examen pienso si las respuestas } \\
\text { fueron las correctas }\end{array}$ & .175 & .370 & .172 & .542 & .100 & .425 & .182 & .717 \\
\hline 14 & $\begin{array}{l}\text { Sé qué estrategias y hábitos de estudio tengo que } \\
\text { cambiar para mejorar y obtener mejores notas }\end{array}$ & .071 & .591 & .007 & .979 & -.002 & .644 & -.006 & 1.00 \\
\hline 17 & $\begin{array}{l}\text { Cuando comienzo una tarea, pienso en las cosas que } \\
\text { ya sé sobre el tema porque eso me ayuda a } \\
\text { comprender mejor }\end{array}$ & .114 & .661 & .037 & .953 & .067 & .701 & .049 & .979 \\
\hline 18 & $\begin{array}{l}\text { Cuando estudio, anoto palabras nuevas, dudas o } \\
\text { ideas importantes }\end{array}$ & -.164 & .67 & -.062 & .905 & -.196 & .681 & -.09 & .865 \\
\hline 20 & $\begin{array}{l}\text { Para mí es importante lograr entender bien las tareas } \\
\text { y el curso }\end{array}$ & .215 & .628 & .097 & .818 & .131 & .692 & .091 & .924 \\
\hline 21 & $\begin{array}{l}\text { Sé cómo utilizar diferentes técnicas y estrategias } \\
\text { para realizar bien mis tareas (como, por ejemplo, } \\
\text { planificar el trabajo, destacar ideas principales, } \\
\text { discutir en grupos, etc.) }\end{array}$ & -.036 & .765 & -.06 & .988 & -.055 & .772 & -.074 & .979 \\
\hline 24 & Después de terminar mis tareas reviso si están bien & .073 & .599 & -.026 & .975 & .041 & .612 & -.034 & .989 \\
\hline 25 & $\begin{array}{l}\text { Cuando finalizo una tarea, pienso si he conseguido } \\
\text { el objetivo que me había propuesto }\end{array}$ & .027 & .833 & -.094 & .98 & -.023 & .869 & -.115 & .973 \\
\hline 26 & $\begin{array}{l}\text { Pongo atención a los comentarios que los profesores } \\
\text { hacen sobre mis trabajos }\end{array}$ & .125 & .513 & .172 & .781 & .048 & .566 & .182 & .854 \\
\hline 3 & Me escapo de clases o me tiro la pera (r) & .178 & -.096 & .739 & .896 & .182 & -.026 & .710 & .907 \\
\hline 4 & Salgo sin pedir permiso del salón (r) & .299 & -.237 & .624 & .594 & & & & \\
\hline 9 & Llego tarde a clases (r) & -.038 & .097 & .377 & .895 & -.057 & .139 & .349 & .772 \\
\hline 11 & $\begin{array}{l}\text { Mis padres o apoderados han sido citados por mi } \\
\text { mala conducta }(r)\end{array}$ & -.094 & .07 & .832 & .971 & -.037 & .053 & .821 & .991 \\
\hline 16 & Me porto bien en clases & -.061 & .300 & .418 & .552 & & & & \\
\hline 23 & Peleo o discuto con mis compañeros en el salón (r) & .051 & -.120 & .726 & .953 & .055 & -.056 & 677 & 980 \\
\hline 28 & $\begin{array}{l}\text { Me mandan a la dirección o al tutor por mi mala } \\
\text { conducta (r) }\end{array}$ & -.231 & .048 & .984 & .919 & -.119 & -.002 & .958 & .977 \\
\hline
\end{tabular}

Nota. AFE=Afectivo; COG=Cognitivo; $\mathrm{CON=Conductual;} \mathrm{(r)=ítem} \mathrm{reverso;} \mathrm{ítem} 29$ fue un caso Heywood (carga factorial >1).

Tabla 5. Índices de bondad de ajuste

\begin{tabular}{lcccc}
\hline & CFI & TLI & RMSEA (IC 90\%) & WRMR \\
\hline Colombia & .983 & .975 & $.052(.044, .060)$ & .817 \\
Uruguay & .986 & .978 & $.043(.030, .055)$ & .603 \\
España & .982 & .973 & $.058(.049, .068)$ & .782 \\
Perú & .988 & .944 & $.035(.025, .044)$ & .660 \\
\hline
\end{tabular}

En primer lugar, se consideraron los índices de ajuste CFI y TLI (>.90; McDonald \& Ho, 2002) y RMSEA (<.08; Jöreskog \& Sörbom, 1993; límite superior del intervalo de confianza <.10; West, Taylo \& Wu, 2012) y la raíz cuadrada media residual ponderada (WRMR <1; DiStefano, Liu, Jiang, \& Shi, 2018). En segundo lugar, se procedió con un enfoque exploratorio basado en la conservación de reactivos siguiendo los siguientes criterios: a) que el ítem tuviera una carga mínima de .40 en su factor teórico; b) que el índice de simplicidad factorial (ISF; Fleming \& Merino, 2005), o el grado en que el ítem fuera influido predominantemente por un solo factor, fuera mayor que .70 (Dominguez-Lara \& Merino-Soto, 2018); y c) en caso de que el ítem tuviera una 
carga factorial mayor que $.40 \mathrm{y}$ un ISF aceptable, y si la magnitud de las cargas secundarias superaban el .30, el ítem se descartaba. Finalmente, la consistencia interna de los cuestionarios fue analizada mediante el alfa de Cronbach, alfa ordinal, y coeficiente omega (>.70; Hunsley \& Marsh, 2008).

\section{Resultados}

Se realizaron los análisis basados en el ESEM por separado con los datos de los cuatro países participantes, repitiéndolos hasta que la solución factorial cumplió con los criterios explicitados. El resultado, considerando los criterios indicados en el apartado de análisis de datos, dio lugar a la retención final de 20 ítems para la validación colombiana (Tabla 1), 16 para la validación uruguaya (Tabla 2), 18 para la validación española (Tabla 3), y 23 para la validación peruana (Tabla 4). Los índices de bondad de los modelos finales fueron adecuados en todos los casos, como se muestra en la Tabla 5.

La fiabilidad medida a través del alfa de Cronbach, el alfa ordinal y el coeficiente omega, fue adecuada en cada uno de los países, y si bien en Uruguay (en el factor afectivo) la fiabilidad no alcanzó el nivel de .70, esta estuvo muy cercana al punto de corte (Tabla 6).

Tabla 6. Coeficientes de fiabilidad

\begin{tabular}{lcccc}
\hline & Colombia & España & Perú & Uruguay \\
\hline Afectivo & & & & \\
Alfa de Cronbach & .79 & .77 & .79 & .64 \\
Alfa Ordinal & .79 & .81 & .80 & .67 \\
Coeficiente omega & .81 & .81 & .81 & .68 \\
Cognitivo & & & & \\
Alfa de Cronbach & .80 & .78 & .86 & .86 \\
Alfa Ordinal & .81 & .82 & .89 & .88 \\
Coeficiente omega & .81 & .83 & .90 & .89 \\
Conductual & & & & \\
Alfa de Cronbach & .89 & .88 & .71 & .71 \\
Alfa Ordinal & .89 & .91 & .82 & .84 \\
Coeficiente omega & .89 & .91 & .84 & .85 \\
\hline
\end{tabular}

\section{Discusión}

Los resultados obtenidos en este estudio muestran en su conjunto la adecuación psicométrica de los cuestionarios para medir el compromiso escolar en cada uno de los cuatro países participantes. El resultado de los análisis factoriales, reveló una estructura similar de tres factores en todos los países, con algunas variaciones en los ítems finalmente retenidos. Respecto a la fiabilidad, el instrumento presentó en los cuatro países, una adecuada consistencia interna en todas sus dimensiones, a excepción de Uruguay, donde el componente afectivo presentó niveles inferiores a .70. No obstante, el valor obtenido fue muy cercano al punto de corte.

Estos datos reflejan que las adaptaciones realizadas en cada país al instrumento chileno original fueron adecuadas, y, por lo tanto, permiten afirmar que se cuenta con un instrumento validado similar para medir el compromiso escolar de estudiantes adolescentes en Chile, Uruguay, España, Perú y Colombia. Este cuestionario permitirá identificar a los y las estudiantes que se encuentran en riesgo de abandono escolar, a partir de la obtención de bajas puntuaciones, indicando además en que componente es más necesario intervenir.

En el caso colombiano, será una herramienta fundamental para la investigación e intervención educativa, pues no existen antecedentes de medidas cuantitativas de este constructo y la mayoría del esfuerzo de las políticas públicas se ha centrado en el acceso a la educación, ignorando una visión integrada de la trayectoria educativa de los y las estudiantes. En los documentos de política pública del país, no se hace ninguna referencia a la importancia del compromiso escolar, ni a su estudio o promoción. En la ley 115 de 1994, que rige la educación en este país, el énfasis se pone en el deber de garantizar el acceso y permanencia de los alumnos y las alumnas al sistema educativo, condición necesaria pero no suficiente para el desarrollo de trayectorias educativas de calidad, por lo que las disposiciones legales posteriores se han centrado principalmente en desarrollar estrategias para la retención de los y las estudiantes más desfavorecidos económicamente, a través de acciones como gratuidad en el transporte y alimentación escolar. Sólo en los documentos de política pública más recientes, aparece la referencia a la necesidad de garantizar trayectorias educativas completas (Ministerio de Educación Nacional de Colombia, 2019), pero con un énfasis aún puesto en la implementación de mecanismos para evitar la deserción, más que en promover que el paso de cada estudiante por el sistema educativo sea una experiencia plena de aprendizaje y desarrollo. 
Hasta la fecha, no se ha podido consolidar un sistema robusto de seguimiento individualizado a las trayectorias escolares, a pesar de que Colombia es uno de los países de la región que más mediciones realiza a sus estudiantes a lo largo de la educación básica (ICFES, 2020). La mayor parte de los informes educativos disponibles, presentan datos generales de los indicadores de deserción, extra edad o repitencia, desperdiciando una oportunidad valiosa de construir una trazabilidad individual de los itinerarios educativos de cada alumno. Aunque es evidente que los programas de apoyo económico son necesarios para facilitar que muchas familias pobres puedan enviar a sus hijos a la escuela, hay evidencias suficientes de que intervenir esta variable, no es suficiente para garantizar trayectorias educativas de calidad (UNESCO, 2020). Por eso, es necesario avanzar hacia una visión integradora de la comprensión del "éxito escolar", a partir de un seguimiento individualizado de los factores individuales, familiares, socioeconómicos y escolares, que afectan el aprendizaje de cada alumno.

En cuanto al caso peruano, el énfasis en la evaluación educativa nacional en educación primaria está centrado en el desempeño en áreas curriculares (matemáticas y comprensión lectora) dejando de lado aspectos motivacionales como se aprecia en la Evaluación Censal de Estudiantes que se desarrolla cada año (Ministerio de Educación, 2019). Con todo, si bien desde el 2007 se desarrolla esta evaluación y los resultados sugieren una mejora continua, lo cierto es que el rendimiento alcanzado por los y las estudiantes en Perú sigue estando por debajo de lo esperado (Guadalupe, Burga, Miranda, \& Castillo, 2015). Esta situación es contraproducente dado que no se abordan aquellos aspectos psicológicos que pueden tener influencia significativa en trayectorias educativa de los estudiantes, sin dejar de lado aquellos determinantes sociales que son igual de importantes, como el aspecto socioeconómico o cultural (Cuenca \& Urrutia, 2019). Por tal motivo, contar con un instrumento de evaluación del compromiso escolar brinda la posibilidad de incluirlo de forma experimental en las evaluaciones $\mathrm{y}$, si los resultados son favorables, podrían ser considerados como política pública y de ese modo analizar su impacto en el rendimiento a gran escala para intervenir según sea necesario.
En el caso de España, las principales investigaciones sobre el compromiso escolar, hasta este momento, se han realizado en regiones del norte y, en la mayoría de los casos utilizando muestras universitarias (Tremols, Fernández, \& Lasarte, 2019). Concretamente en población adolescente, contamos con tres antecedes recientes que indagan sobre el estudio del compromiso escolar (Fernández, Revuelta, Maya, \& Lasarte, 2018; Goñi, Ros, \& Fernández-Lasarte, 2018; Suarez et al., 2019), lo que denota que se trata de un concepto emergente en el panorama académico español, que aún no ha tenido un correlato claro en el ámbito de las políticas públicas. No obstante, resulta relevante que el reciente anteproyecto de ley educativa en España (LOMLOE, 2020) mencione, en su exposición de motivos, a la idea (ya expresada en la Ley Educativa de 2006, que retoma) de que, para "garantizar una educación de calidad para todos los ciudadanos es imprescindible el compromiso de todos los componentes de la comunidad educativa y del conjunto de la sociedad" y que "una de las consecuencias más relevantes del principio del esfuerzo compartido consiste en la necesidad de llevar a cabo una escolarización equitativa del alumnado" (p. 2). Estas apreciaciones parecen conectar con una perspectiva amplia, defendida en la presente investigación, sobre el compromiso escolar, que va más allá del compromiso como un rasgo del estudiante y que lo concibe como un "estado" (Lara et al., 2018) que, si bien inevitablemente toma a aquel como "unidad de observación", parte de una unidad de análisis (multidimensional) mucho más amplia.

En el caso de Uruguay, en 2015 la Administración Nacional de Educación Pública de Uruguay creó el Sistema de Protección de Trayectorias Educativas (SPTE), con el cometido de dar seguimiento a las trayectorias escolares, detectar situaciones de vulnerabilidad educativa e intervenir en situaciones de riesgo de abandono escolar. Si bien el SPTE priorizó la generación de sistemas informáticos de seguimiento, alerta temprana y protección de las trayectorias estudiantiles en el marco de un proceso sistemático y planificado orientado a la retención en el sistema educativo de los y las estudiantes más vulnerables, el fomento del compromiso 
escolar, tal como se lo entiende aquí, no tuvo una presencia explícita en sus acciones. Algo similar puede plantearse con respecto al vasto repertorio de programas educativos implementados por el Estado uruguayo desde mediados de la primera década del presente siglo. Entre los programas que se implementaron para la educación media, los más destacables son los siguientes: Tránsito Educativo (focalizado en la transición entre educación primaria y educación media), el programa de Impulso a la Universalización del Ciclo Básico (focalizado en la prevención del abandono y la repetición de la población más vulnerable), Compromiso Educativo (orientado a la retención y continuación en educación media superior), Aulas Comunitarias (en procura de la reinserción de estudiantes de 12 a 15 años que abandonaron sus estudios o que presentan riesgos de hacerlo), el programa de Acompañamiento Pedagógico a la Trayectoria Estudiantil y el programa Liceos con Tutorías (Acevedo \& Menni, 2016). En este contexto, y sobre la base de todo el desarrollo precedente, la implementación de programas orientados al fomento del compromiso escolar, si están sustentados en estudios empíricos sólidamente fundados, contribuirán en forma significativa a una mayor persistencia en educación media y, sobre esa base, al éxito estudiantil en su sentido más cabal.

En conclusión, este estudio ha permitido contar con un cuestionario para medir el compromiso escolar adaptado y validado para cada país, permitiendo por lo tanto tener una herramienta valiosa para detectar a los y las estudiantes que se encuentran en riesgo de deserción social, así como para promover a las trayectorias educativas exitosas (Christenson et al., 2008). Como futuras líneas de investigación, sería adecuado adaptar y validar el cuestionario en otros países iberoamericanos que se encuentran en situaciones similares y a los que contar con un instrumento de estas características les permitiría también avanzar en la promoción de estas trayectorias educativas. En esta línea, recientemente contamos con el trabajo realizado en Argentina (Batistelli, 2020), donde realizan una primera adaptación del CCE a través del uso de mapas. Este trabajo abre la posibilidad de tratar con los aspectos relacionados con el compromiso escolar que se encuentran detrás de la repetición y fracaso escolar encontrados en dicho país (Cayssials, 2005).

\section{Financiamiento}

Este artículo ha recibido el apoyo de los proyectos FONDEF ID14I20078 y FONDEF IT 19I0012 financiados por la Comisión Nacional de Investigación Científica y Tecnológica CONICYT, Chile

\section{Referencias}

Acevedo, F. (2020). Factores explicativos del abandono de los estudios en la educación superior en contextos socio-académicos desfavorables. Revista Española de Pedagogía, 78(276), 253-269. doi:10.22550/REP78-2-2020-02

Acevedo, F., \& Menni, G. (2016). Programas de inclusión educativa implementados en la Educación Media pública de Uruguay: Breve estado del arte y una mirada hacia el programa Compromiso Educativo. En Dalla Corte y Carneiro Sarturi (Eds.), Políticas Públicas da Educação Básica e Superior. Santa Maria: Universidade Federal de Santa Maria. RS, Brasil.

ANEP-CODICEN (Administración Nacional de Educación Pública - Consejo Directivo Central). (2013). Uruguay en PISA 2012. Primeros resultados en Matemática, Ciencias y Lectura del Programa Internacional de Evaluación de Estudiantes, PISA de OCDE. Informe Preliminar. Montevideo: inédito.

Appleton, J. J., Christenson, S. L., \& Furlong, M.

J. (2008). Student engagement with school: Critical conceptual and methodological issues of the construct. Psychology in the Schools, 45(5), 369-386. doi:10.1002/pits.20303

Asparouhov, T., \& Muthén, B. (2009). Exploratory structural equation modeling. Structural Equation Modeling: A Multidisciplinary Journal, 16(3), 397-438. doi:10.1080/10705510903008204

Banco mundial. (2020). Datos de libre acceso. Colombia. Recuperado de https://datos.bancomundial.org/pais/colombia ?view $=$ chart

Batistelli, M. (2020). El uso de mapas de compromiso escolar para una educación 
personalizada (Tesis de maestría). Universidad Austral, Argentina. Recuperada de

https://riu.austral.edu.ar/handle/123456789/87 5

Cayssials, A. N. (2005). Evaluación de atribuciones de causalidad en adolescentes repetidores. Revista Iberoamericana de Diagnóstico y Evaluación - e Avaliação Psicológica, 1(19), 79-88. Recuperado de https://www.redalyc.org/articulo.oa?id=45 9645450005

Christenson, S. L., Reschly, A. L., Appleton, J. J., Berman, S., Spanjers, D. M., \& Varro, P. (2008). Best practices in fostering student engagement. En A. Thomas y J. Grimes (Eds.), Best practices in school psychology $V$, vol. 6 (pp. 1099-1120). Bethesda, MD, EE.UU.: National Association of School Psychologists.

Cotler, J. (2011). Las desigualdades en el Perú: balances críticos. Lima, Perú: Instituto de Estudios Peruanos.

Cuenca, R., \& Urrutia, C. (2019). Explorando las brechas de desigualdad educativa en el Perú. Revista Mexicana de Investigación Educativa, 24(81), 431-461.

De Armas, G. (2017). Trayectorias educativas en Uruguay. Principales rasgos, tendencias y desafios para las políticas públicas. Montevideo: UNICEF. Recuperado de https://www.bibliotecaunicef.uy/doc_num.php ?explnum_id=184

De Armas, G., \& Retamoso, A. (2010). La universalización de la educación media en Uruguay. Tendencias, asignaturas pendientes y retos a futuro. Montevideo: UNICEF.

DiStefano, C., Liu, J. Jiang, N., \& Shi, D. (2018). Examination of the weighted root mean square residual: Evidence for trustworthiness?. Structural Equation Modeling: $\quad A \quad$ Multidisciplinary Journal, 25(3), 453-466, doi:10.1080/10705511.2017.1390394

Dominguez-Lara, S., \& Merino-Soto, C. (2018). Estructura interna del BFI-10P y BFI-15P: un estudio complementario con enfoque CFA y ESEM. Revista Argentina de Ciencias del Comportamiento, 10(3), 22-34. doi:10.32348/1852.4206.v10.n3.21037
Enguita, M. F. (2011). Del desapego al desenganche y de este al fracaso. Revista de Sociología de la Educación-RASE, 4(3), 255269.

Fernández, A. R., Revuelta, L. R., Maya, M. S., \& Lasarte, O. F. (2018). The role of parental socialization styles in school engagement and academic performance. European Journal of Education and Psychology, 11(2), 123-139.

Fleming, J., \& Merino, C. (2005). Medidas de simplicidad y ajuste factorial: Un enfoque para la construcción y revisión de escalas derivadas factorialmente. Revista de Psicología, 23(2), 252-266. Recuperado de http://revistas.pucp.edu.pe/index.php/psicolog $\mathrm{ia} /$ article/view/2150

Fredricks, J. A. (2014). Eight myths of student disengagement: Creating classrooms of deep learning. Thousand Oaks, CA, EE.UU.: Corwin.

Fredericks, J. A., Blumenfeld, P., Friedel, J., \& Paris, A. (2005). School engagement, What do children need to flourish?: Conceptualizing and measuring indicators of positive development. New York, NY: Springer Science and Business Media.

Fredricks, J. A., \& McColskey, W. (2012). The measurement of student engagement: A comparative analysis of various methods and student self-report instruments. En S. L. Christenson, A. L. Reschly y C. Wylie (Eds.), Handbook of research on student engagement (pp. 763-782). Nueva York, NY, EE.UU.: Springer. doi: 10.1007/978-1-4614-2018-7_37

Gelber, D. (2010). Trayectorias de riesgo, éxito y abandono en Ciclo Básico en Uruguay. Páginas de Educación, 3(1), 61-81. doi: 10.22235/pe.v3i1.658

Guadalupe, C., Burga, A., Miranda, L., \& Castillo, L. (2015). Brechas de equidad en la evaluación censal de estudiantes 2007-2014: tres aproximaciones a su medición. Persona, 18, 47-68. Recuperado de http://revistas.ulima.edu.pe/index.php/Persona /article/view/498

Goñi, E., Ros, I., \& Fernández-Lasarte, O. (2018). Academic performance and school engagement among secondary school students in accordance with place of birth, gender and 
age. European Journal of Education and Psychology, 11(2), 93-105. doi:10.30552/ejep.v11i2.224

Gutiérrez, M., Tomás, J. M., Gómez, M., \& Moll, A. (2019). Clima motivacional, satisfacción, compromiso y éxito académico en estudiantes angoleños y dominicanos. Psicologia Escolar e Educacional, 23, 1-9. doi:10.1590/2175-35392019018764

Hazel, C. E., Vazirabadi, G. E., \& Gallagher, J. (2013). Measuring aspirations, belonging, and productivity in secondary students: Validation of the Student School Engagement Measure. Psychology in the Schools, 50(7), 689-704. doi:10.1002/pits.21703

Hunsley, J., \& Marsh, E.J. (2008). Developing criteria for evidence-based assessment: An introduction to assessment that work. En J. Hunsley y E. J. Marsh (Eds.) A guide to assessments that work (pp. 3-14). Oxford: Oxford University Press.

Instituto colombiano para la evaluación de la Educación -ICFES- (2020). SABER $3^{\circ}, 5^{\circ}, 9^{\circ}$ $Y 11^{\circ}$. Síntesis de resultados. Recuperado de https://www.icfes.gov.co/documents/20143/2 34129/Informe+Saber+3+5++9 y11+Sintesis+de+resultados2020.pdf

Jöreskog, K. G., \& Sörbom, D. (1993). LISREL 8: Structural equation modeling with the SIMPLIS command language. Scientific Software International; Lawrence Erlbaum Associates, Inc.

Lam, S.-f., Jimerson, S., Wong, B. P. H., Kikas, E., Shin, H., Veiga, F. H., ... \& Zollneritsch, J. (2014). Understanding and measuring student engagement in school: The results of an international study from 12 countries. School Psychology Quarterly, 29, 213-232.

Lara, L., Saracostti, M., Navarro, J. J., de-Toro, X., Miranda-Zapata, E., Trigger, J. M., \& Fuster, J. (2018). Compromiso escolar: Desarrollo y validación de un instrumento. Revista Mexicana de Psicología, 35(1), 52-62.

Latorre, M. (2018). Fracaso escolar en estudiantes de educación secundaria de colegios públicos de Perú, 2005-2010, y sus factores asociados. Revista EDUCA UMCH, 11, 13-50. doi:10.35756/educaumch.v11i0.67

LOMLOE, Anteproyecto de ley. 13 de marzo de 2020. Boletín Oficial de las Cortes Generales.
Recuperado

https://www.educacionyfp.gob.es/dam/jcr:8c7 d3863-aaa7-4bbd-91b2-4d05bcb80286/apllomloe-web2020-03-03.pdf

McDonald, R. P., \& Ho, M.-H. R. (2002). Principles and practice in reporting structural equation analyses. Psychological Methods, 7, 64-82. doi:10.1037/1082-989X.7.1.64

Ministerio de Educación. (2019). Resultados de la Evaluación Censal de Estudiantes 2019. Lima: Autor. Recuperado de http://umc.minedu.gob.pe/ece2019/

Ministerio de Educación Nacional de Colombia. (2019). Decreto 2370 de 27 de diciembre de 2019. Recuperado de

https://dapre.presidencia.gov.co/normativa/no rmativa/DECRETO $\% 202370 \% 20 \% 20$ DEL $\%$ 2027\%20DICIEMBRE\%20DE\%202019.pdf

Ministerio Educación y Formación Profesional. (2017). Enseñanzas no universitarias, curso 2016-17. Resultados detallados.

Miranda-Zapata, E., Lara, L. Navarro, J.J., Saracostti, M., \& de-Toro, X. (2018). Modelización del efecto del compromiso escolar sobre la asistencia a clases y el rendimiento escolar. Revista de Psicodidáctica, 23(2), 102-109. doi:10.1016/j.psicod.2018.02.003.

Muelle, L. (2016). Factores de riesgo en el bajo desempeño académico y desigualdad social en el Perú según PISA 2012. Apuntes, 43(79), 945. doi:10.21678/apuntes.79.865

Muthén, L. K., \& Muthén, B. O. (1998-2015). Mplus User's guide (7th ed.). Los Angeles, CA: Muthén \& Muthén.

Nie, Y., \& Lau, S. (2009). Complementary roles of care and behavioral control in classroom management: The self-determination theory perspective. Contemporary Educational Psychology, 34, 185-194.

doi:10.1016/j.cedpsych.2009.03.001

Organización para la Cooperación y el Desarrollo Económico, OCDE. (2007). Understanding the Social Outcomes of Learning. OECD Publishing.

Organización para la Cooperación y el Desarrollo Económico, OCDE. (2016). Programa para la Evaluación Internacional de Alumnos (PISA). París: Organización para la Cooperación y el Desarrollo Económicos. 


\section{Recuperado}

de https://www.oecd.org/pisa/data/2015databa se/

Organización para la Cooperación y el Desarrollo Económico, OCDE. (2019). Programa para la Evaluación Internacional de Alumnos (PISA). París: Organización para la Cooperación y el Desarrollo Económicos. Recuperado de https://www.oecd.org/pisa/data/2018databa $\mathrm{se} /$

Pineda-Báez, C., Hennig Manzuoli, C., \& Vargas Sánchez, A. (2019). Supporting student cognitive and agentic engagement: Students' voices. International Journal of Educational Research, 96, 81-90.

Programme for International Student Assessment. PISA. (2018). Microdatos. OCDE.

Politikon (2017). El muro invisible: Las dificultades de ser joven en España. Debate.

Rodríguez-Fernández, A., Ramos-Díaz, E., Fernández-Zabala, A., Goñi, E., Esnaola, I., \& Goñi, A. (2016). Contextual and psychological variables in a descriptive model of subjective well-being and school engagement. International Journal of Clinical and Health Psychology, 16(2), 166-174. doi:10.1016/j.ijchp.2016.01003

Saracostti, M., Lara, L., \& Miranda-Zapata, E. (2019). Technological platform for online assessment and report generation of school engagement and contextual factors: Brief technical report. Electronic Journal of Research in Educational Psychology, 17, 193212.

Sinatra, G. M., Heddy, B. C., \& Lombardi, D. (2015). The challenges of defining and measuring student engagement in science. Educational Psychologist, 50(1), 1-13. doi:10.1080/00461520.2014.1002924

Suárez, N., Regueiro, B., Estévez, I., del Mar Ferradás, M., Guisande, M. A., \& Rodríguez, S. (2019). Individual precursors of student homework behavioral engagement: The role of intrinsic motivation, perceived homework utility and homework attitude. Frontiers in Psychology, 10, 941 . doi:10.3389/fpsyg.2019.00941

Tarabini, A., Curran, M., Montes, A., \& Parcerisa, L. (2018). Can educational engagement prevent early school leaving? Unpacking the school's effect on educational success. Educational Studies, 45(2), 226-241. doi:10.1080/03055698.2018.1446327

Tremols, I., Fernández, A., \& Lasarte, O. (2019). Autoconcepto académico, motivación escolar e implicación escolar del alumnado universitario de República Dominicana y España. Aula Abierta, 48(3), 271-278. doi:10.17811/rifie.48.3.2019.271-278

UNESCO. (2020). Informe de seguimiento de la educación en el mundo, 2020: Inclusión y educación: todos sin excepción. Paris: UNESCO. Recuperado de https://gem-report2020.unesco.org/es/inicio/

Veiga, F. H. (2016). Assessing student Engagement in School: Development and validation of a four-dimensional scale. Procedia-Social and Behavioral Sciences, 217, 813-819. doi:10.1016/j.sbspro.2016.02.153

Wang, M.-T., Fredricks, J., Ye, F., Hofkens, T., \& Linn, J. S. (2019). Conceptualization and assessment of adolescents' engagement and disengagement in school: A Multidimensional School Engagement Scale. European Journal of Psychological Assessment, 35(4), 592-606. doi:10.1027/1015-5759/a000431

West, S. G., Taylor, A. B., \& Wu, W. (2012). Model fit and model selection in structural equation modeling. En R. H. Hoyle (Ed.), Handbook of Structural Equation Modeling (pp. 209-231). New York: The Guilford Press.

Zaff, J. F., Donlan, A., Gunning, A., Anderson, S. E., McDermott, E., \& Sedaca, M. (2017). Factors that promote high school graduation: A review of the literature. Educational Psychology Review, 29(3), 447-476. doi:10.1007/s10648-016-9363-5 